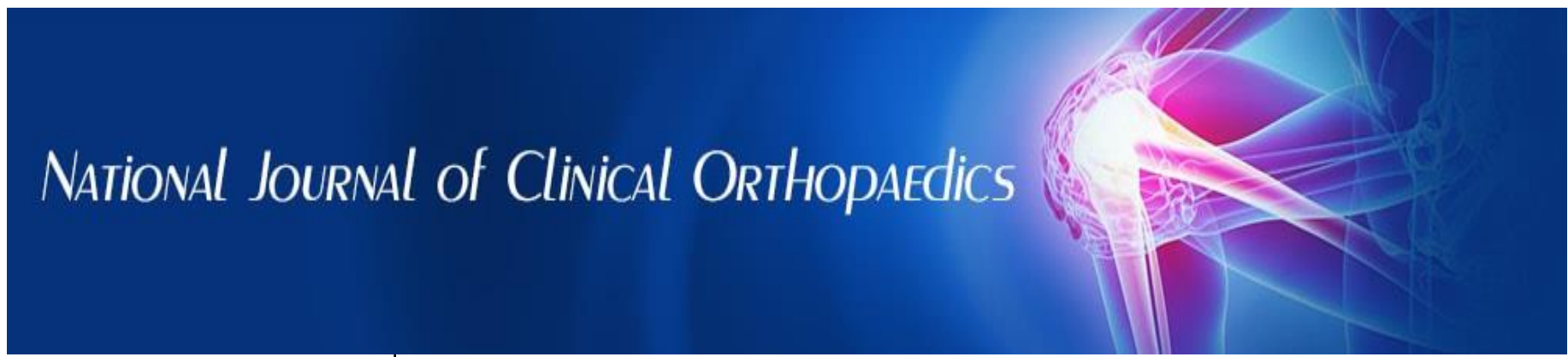

ISSN (P): 2521-3466

ISSN (E): 2521-3474

(C) Clinical Orthopaedics

www.orthoresearchjournal.com

2019; 3(2): 22-25

Received: 11-02-2019

Accepted: 15-03-2019

Dr. Sravya Teja Paleti

Assistant Professor, Dept. of

orthopaedics ASRAM medical

College, Malkapuram, West

Godavari, Andhra Pradesh,

India

Dr. Satish M

Consultant Orthopaedician,

My cure Hospitals, Vizag,

Andhra Pradesh, India
Correspondence

Dr. Sravya Teja Paleti

Assistant professor, Dept. of orthopaedics ASRAM medical College, Malkapuram, West Godavari, Andhra Pradesh, India

\section{Role of negative pressure wound therapy in orthopaedics: Our experience in 20 cases}

\author{
Dr. Sravya Teja Paleti and Dr. Satish M
}

DOI: $\underline{\text { https://doi.org/10.33545/orthor.2019.v3.i2a.05 }}$

\section{Abstract}

Background: Open injuries and infections are still the concern for the orthpadecians. Negative pressure wound therapy is one of several methods enabling to obtain better treatment results in case of open infected wounds. The use of negative pressure therapy enables to obtain a reduction in the number of bacteria which significantly reduces the number of complications and helps to decrease th wound size significantly.

Aims and Objectives: To review the usefulness of negative pressure wound therapy in Orthopaedics.

Materials and Methods: The cases presented in this study are those who were admitted in King George Hospital in the time period from September 2013 to September 2016. This is a prospective interventional study. In this study, 20 patients were assigned to the study group (Negative Pressure Wound TherapyNPWT) based on their willingness for undergoing treatment.

Observations and Results: 12 males and 8 females are involved in the study. There is decrease in the mean wound area from $64 \mathrm{~cm} 2$ to $38 \mathrm{~cm} 2$. There is decrease in the duration of hospital stay. Finally, wound is closed by SSG or secondary suturing.

Discussion: NPWT is known to decrease bacterial counts. NPWT significantly decreases infection rates, enhances wound healing there by decreases the hospital stay to the patients. In our series, we had $70 \%$ (14 cases) excellent results and 30\% (6 cases) good results and no poor results. As interpretation with results, VAC therapy is effective mode of adjuvant therapy for the management of infected wounds.

Conclusion: VAC has been proven to be a reliable method of treating a variety of infected wounds. It greatly increases the rate of granulation tissue formation and lowers bacterial counts to accelerate wound healing. It can be used as a temporary dressing to prepare wounds optimally prior to closure or as a definitive treatment for nonsurgical and surgical wounds. VAC is now being used in a multitude of clinical settings, including the treatment of surgical wounds, infected wounds, traumatic wounds, pressure ulcers, diabetic foot ulcers and venous stasis wounds, amputation stumps.

Keywords: VAC, NPWT, Chronic osteomyelitis, chronic wounds, infected wounds

\section{Introduction}

Large, complicated wounds pose a significant surgical problem. The duration of treatment is usually long, the number of complications and therapy failures are significant, and one should not forget about high treatment cost and long hospital stay. NPWT is appliying constant subatmospheric pressure on the wound which effectively removes excess of interstitial fluid from wound, thereby reducing bacterial proliferation and wound infection. Negative pressure wound therapy is one of several methods enabling to obtain better treatment results in case of open infected wounds ${ }^{[1,2]}$. We here share with you our experience of NPWT in orthopaedic department.

\section{Aims and Objectives}

To review the Role of NPWT in wound healing in orthopaedics. The primary efficacy end point is the complete wound closure. Secondary end points included reduction in the wound surface area over time, time to achieve complete wound closure either by surgery or secondary intention, and a reduction in complications, including amputations.

\section{Materials and Methods}

The cases presented in this study are those who were admitted as in patients at King George Hospital in the time period from September 2013 to September 2016. This is a prospective 
interventional study. In this study, 15 patients were assigned to the study group (Negative Pressure Wound Therapy) based on their willingness for undergoing treatment.

Inclusion criteria: Post-traumatic cases of infected open wounds presented to the hospital during the study period.

- Post-operative infected wounds.

- Non-healing wounds.

- Delayed healing wounds.

- Skin grafts

- Amputation stumps

\section{Exclusion criteria}

- Chronic osteomyelitis.

- Malignancy.

- Raw area with exposed vessels and nerves.

- Patients not willing for VAC therapy.

\section{Imaging studies}

- X-ray of the affected limb.

- $\quad$ MRI of affected part if needed.

- Culture and sensitivity of the pus/discharge from the ulcer.

\section{Procedure}

After evaluating the patient and under aseptic precautions, wound was debrided thoroughly and skin was cleaned around the wound and kept dry. Wound debridement was done after confirmation of diagnosis (Figure 1). Regular dressings were done once in 2 days basis. Granu Foam Dressing (Black Foam) was used in NPWT. It has been specifically engineered to deliver NPWT. It has pore size of 400-600 microns. It is hydrophobic. Open pore structure of VAC. Granu Foam Dressings adapt to the contours of deep or irregularly shaped wounds in order to provide equal distribution of pressure at the wound site. This is autoclavable and has requisite softness at the applied negative pressure of about $150 \mathrm{mmHg}$ (Figure 2). The foam is cut to the shape of the wound.

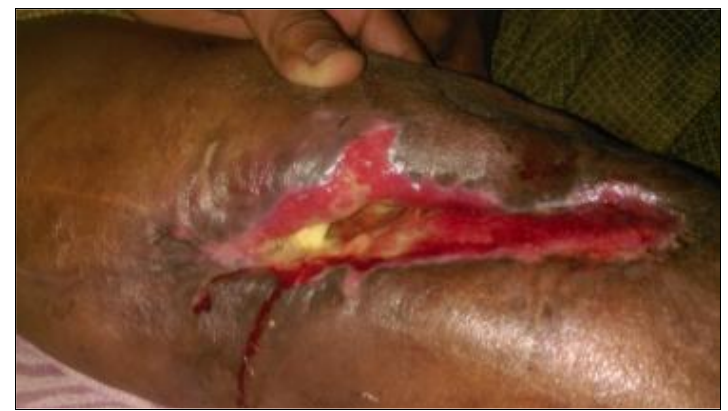

Fig 1: Wound before VAC Appli Cation

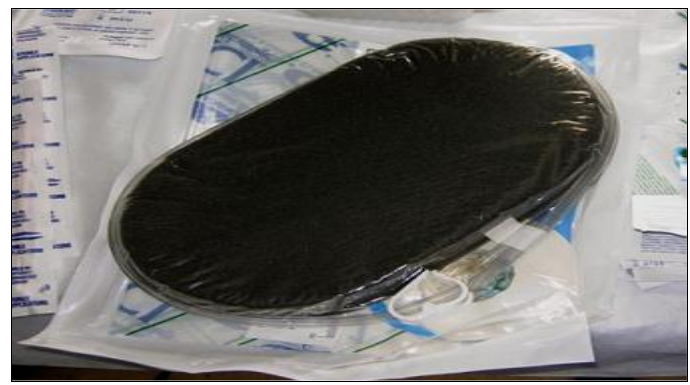

Fig 2: Granu foam dressing black foam
Foam is placed over the wound and transparent drape material applied to cover the foam and wound. The drape material should extend out onto the peri wound about $5 \mathrm{~cm}$ all around. (Figure 4)

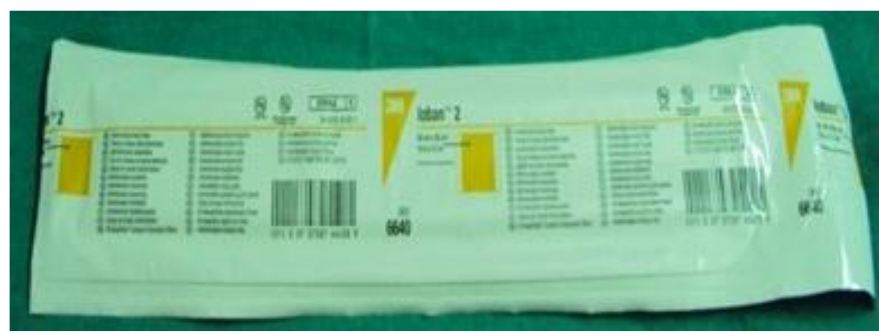

Fig 3: Adhesive Steridrape

The suction tube passed under the adhesive Steridrape. The area where the tubing enters is prone to leaks and hence care must be taken to seal that area properly. (Figure 4). Tubing connected from dressing to tubing coming from VAC suction apparatus. The settings were adjusted in the VAC machine. The pressure adjusted to negative suction of $125-150 \mathrm{mmHg}$. Intermittent negative pressure was applied as machine is turned on for 5 minutes and off for 3 minutes. Upon application of the suction, the foam collapses into the wound and the negative pressure absorbs the fluid through the wound

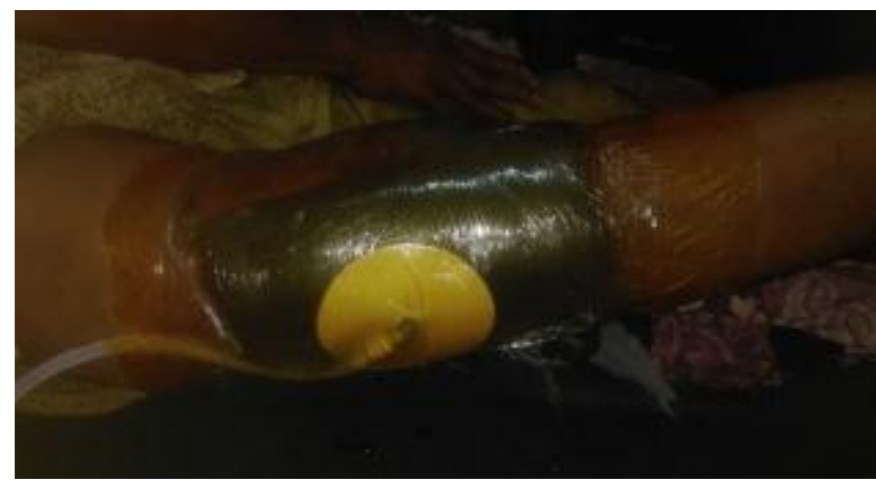

Fig 4: After VAC Dressing

Dressing change was done once in $48 \mathrm{hrs}$. irrespective of the wound condition. Evidence of wound healing was confirmed by the amount of granulation tissue, culture and sensitivity, CRP and clinical findings. The initial size of the wound before placement of VAC was approximately calculated by placing a transparent sheet as close to the wound as possible. The edges of the wound were traced over the transparent sheet (Figure 5). The transparent sheet containing the wound imprint was placed over a $1 \mathrm{~cm} 2$ graph paper. The number of half squares, squares which were more than half and full squares were counted which determines the size of the wound
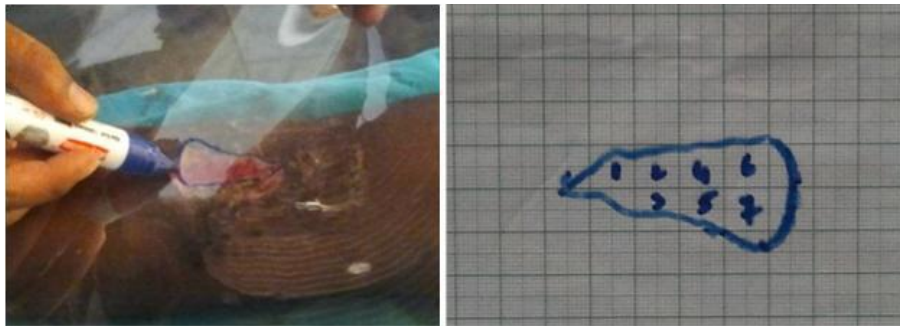

Fig 5: Measuring the wound

Once the wound showed evidence of healing or good granulation tissue, a split skin grafting or secondary suturing of 
the wound was carried out. Cases were followed 10 days after discharge.

\section{Observations and Results}

20patients aged between 16-72 years of age group with infected wounds treated with VAC therapy. 12 males and 8 females are included in the study. Large number of cases in our study comprise of infected wounds of thigh ( 7 cases, 35\%), leg (8 cases, $40 \%$ ), back ( 2 cases, $10 \%$ ) and foot ( 3 cases, $15 \%$ ). In our study, VAC dressing was applied for 6 days (8 cases, 40\%), 8 days ( 7 cases, 35\%), 4 days ( 2 cases, 10\%), 10 days ( 3 cases, $15 \%$ ). 60 (12) of the cases required less than 4 dressings and 40
(8) of the cases required more than 4 dressings for wound closure. Duration of stay in hospital was less than 1 month in $35 \%$ (7) of the cases and more than 1 month in $65 \%$ (13) of the cases. Mean wound size was $64 \mathrm{~cm} 2$ before the application of VAC dressing which reduced to a mean of $38 \mathrm{~cm} 2$ at the completion of VAC therapy. Wound closure was achieved by secondary suturing in 50\% (10) of the cases and split skin grafting in $50 \%$ (10) of the cases. Wound healing was excellent in $70 \%$ (14) of the cases and good in 30 (6) of the cases.

\section{Case 1}

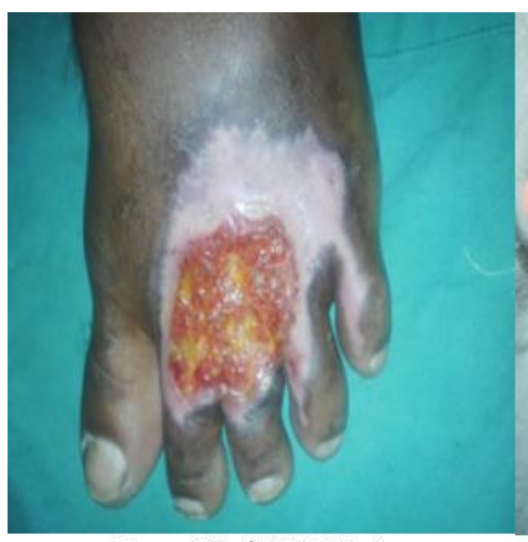

Open \# 2nd MT Lt Foot

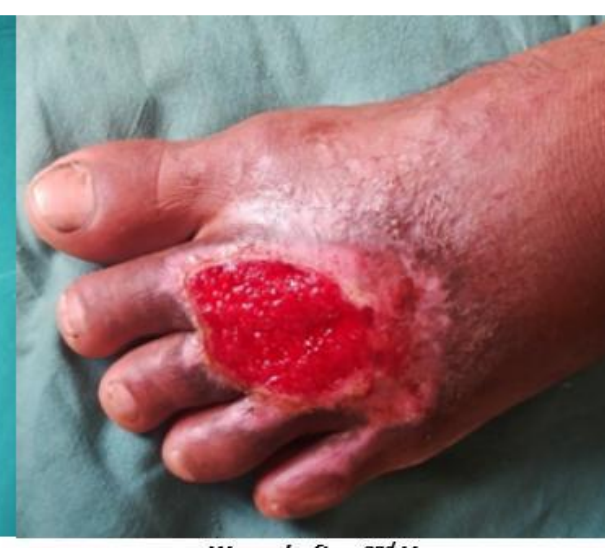

Wound after $2^{\text {nd }}$ Vac

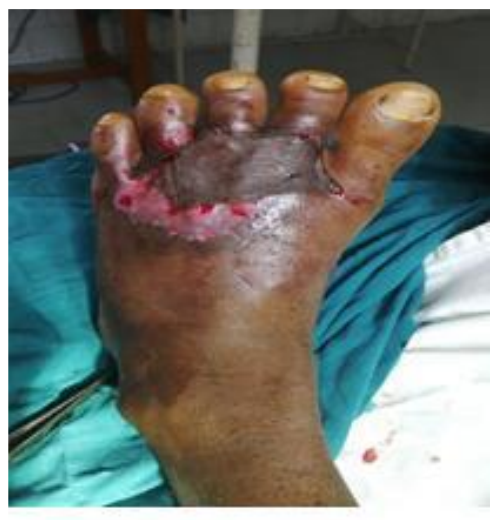

Wound closed with SSG

Case 2

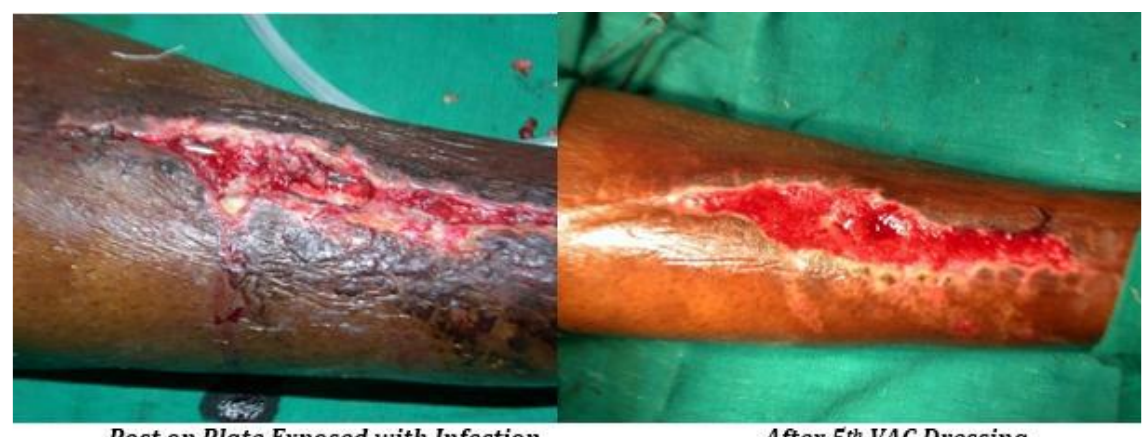

Post op Plate Exposed with Infection

After $5^{\text {th }}$ VAC Dressing

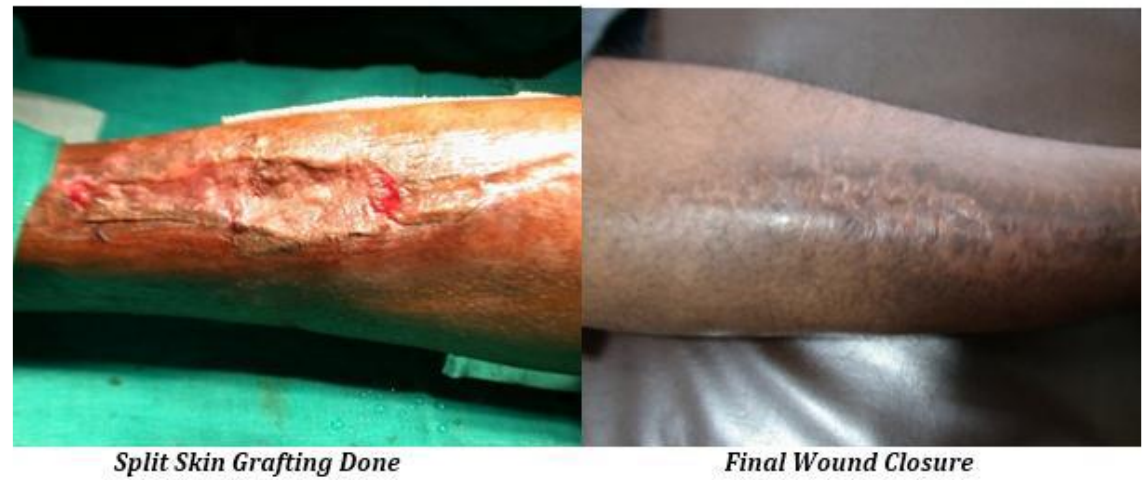

\section{Discussion}

The pathogenesis of infection in fractures fixation devices is related to micro-organisms, which grow in biofilm, and therefore its eradication is difficult ${ }^{[3]}$. There are various mechanisms by which NPWT facilitates wound healing ${ }^{[4]}$. It facilitates fluid removal from the wound, thereby reducing the wound size. By increasing the blood flow, NPWT helps early granulation tissue formation NPWT known to reduce bacterial counts, although remain colonised with organisms. ${ }^{5}$ Wounds covered with NPW dressing are completely isolated from the environment, thereby reducing cross infection. Application of negative pressure wound therapy causes changes in the tissues in the vicinity of its application. As interpretation with results, VAC therapy is effective mode of adjuvant therapy for the management of infected wounds.

\section{Conclusion}

VAC has been proven to be a reliable method of treating a variety of infected wounds. It greatly increases the rate of granulation tissue formation and lowers bacterial counts to 
accelerate wound healing. It can be used as a temporary dressing to prepare wounds optimally prior to closure or as a definitive treatment for nonsurgical and surgical wounds. The VAC device is well tolerated with few complications or contraindications and is playing an ever-expanding role in wound care. The limitation of this study is that this was a single-arm, prospective, observational study with no control group. Comparison to historical data is often difficult since different criteria are being used and no simple one-to-one comparison is possible. One more limitation is a short followup period.

\section{References}

1. Hunter JE, Teot L, Horch R et al. Evidence based medicine: vacuum assisted closure in wound care management. Int Wound J. 2007; 4(3):256-269.

2. Jerome D. Advances in negative pressure wound therapy: the VAC instill. J Wound Ostomy Continence Nurs. 2007; 34(2):191-194

3. Trampuz A, Zimmerli W. Diagnosis and treatment of infections associated with fracture-fixation devices. Injury. 2006; 37(2):S59-66.

4. Argenta LC, Morykwas MJ. Vacuum assisted closure: a new method for wound control and treatment: clinical experience. Annals of Plastic Surg. 1997; 38(6):563-576.

5. Kucan JO, Robson MC, Heggers JP et al. Comparison of silver sulfadiazine, povidone-iodine and physiologic saline in the treatment of chronic pressure ulcers. J Am Geriat Soc. 1981; 29(5):232-235. 